\title{
THE LONG PERIOD DWARF NOVA UY PUP IN OUTBURST
}

\author{
J. J. LOCKLEY ${ }^{1}$, JANET H. WOOD ${ }^{1}$, D. H. P. JONES ${ }^{2}$, \\ S. MINESHIGE \\ 1. Physics Department, Keele University, Keele, ST5 5BG, UK \\ 2. Royal Greenwhich Observatory, Madingley Road, \\ Cambridge, CBз OEZ, UK \\ 3. Dept. of Astronomy, Kyoto University, Sakyo-Ku, \\ Kyoto 606-01, Japan
}

\section{Introduction}

UY Pup is a little studied, frequently outbursting dwarf nova whose brightness varies from $V \sim 13.5 \mathrm{mag}$ in outburst to $V \sim 15.8 \mathrm{mag}$ in quiescence, which typically lasts $\sim 15 \ldots 25 \mathrm{~d}$. We observed it spectroscopically at the INT in La Palma on 1989 Febuary 15 and 16 during outburst maximum. In this outburst, the rise lasted at least $3 \mathrm{~d}$ and there was a long decline of about $6 \ldots 9 \mathrm{~d}$. The whole outburst lasted about $18 \mathrm{~d}$ (Mattei, private communication).

\section{Results}

$\mathrm{H} \alpha, \mathrm{H} \beta$ and $\mathrm{He}$ I $(6678 \AA)$ lines are clearly present. The $\mathrm{H} \alpha$ emission line is superimposed on a very weak absorption component. In $\mathrm{H} \beta$, however, the emission is very weak and the absorption component stronger. The emission components of the $\mathrm{H} \alpha$ and $\mathrm{H} \beta$ lines both have FWZI $\sim 925 \mathrm{~km} \mathrm{~s}^{-1}$, whereas the absorption around $\mathrm{H} \beta$ has FWZI $\sim 4600 \mathrm{~km} \mathrm{~s}^{-1}$. These lines are typical of Balmer lines seen in low inclination, high mass transfer rate systems, both nova-like variables and other dwarf novae at the same stage of outburst. Theoretical models with mass transfer rates of $\dot{M}=(1 \ldots 3) 10^{16} \mathrm{~g} \mathrm{~s}^{-1}$ and orbital inclination $i=15^{\circ}$ have very similar profiles to the $\mathrm{H} \alpha$ and $\mathrm{H} \beta$ profiles in UY Pup [see Fig. 5i-j in Cheng \& Lin (1989)]. Therefore Balmer lines of the type seen in UY Pup can be produced by discs alone. We find no evidence for a second component to the line, from, for example, irradiation of the secondary star. There is variation by a factor of 2 in the equivalent width of $\mathrm{H} \alpha$, though this does not appear to be related to the orbital cycle or the evolution through the outburst. 
Radial velocities were measured from $\mathrm{H} \alpha$ using the double Gaussian method (Shafter 1983). The resulting radial velocities gave a best fit orbital period of $0.423 \pm 0.003 \mathrm{~d}$, however a period of $0.297 \pm 0.001 \mathrm{~d}$ was also found. From Monte Carlo simulations of radial velocity data sets generated at both periods we concluded that, while the longer of the two periods remains the most likely, our sampling would generate an alias at the longer period if the shorter one were real. Our best fit parameters are $P=0.423 \mathrm{~d}, K_{\mathrm{em}}=$ $24.5 \pm 8.5 \mathrm{~km} \mathrm{~s}^{-1}, \gamma=54.8 \pm 3.5 \mathrm{~km} \mathrm{~s}^{-1}$ and $T_{0}=2447574.072 \pm 0.006 \mathrm{~d}$. If this is the true period, then UY Pup has one of the longest periods of all dwarf novae. With $P=0.423 \mathrm{~d}$, the relations given by Szkody \& Mattei (1984) predict an outburst duration of $17.6 \mathrm{~d}$ and a decline of $7.9 \mathrm{~d}$, in good agreement with the outburst of UY Pup that we observed.

\section{Estimation of the system parameters}

Assuming the longer period is correct and that the secondary is a main sequence star, given a value for the mass ratio, $q$, we can calculate the primary mass, the primary radius, the orbital separation and the radius of the secondary. We can then place limits on $q$ by comparing the FWZI of the emission lines with the Keplerian velocity of material at the primary surface and using the upper mass limit of the primary star. We also consider that the secondary may be evolved from the main sequence (Friend et al. 1990 and references therein) and re-calculated the parameters assuming a secondary mass only half that of the main sequence mass. Both sets of parameters are listed in Table 1.

TABLE 1. Estimated Binary Parameters

\begin{tabular}{lll}
\hline & Main Sequence Secondary & Non Main Sequence Secondary \\
\hline Secondary Mass & $1.12 \mathrm{M}_{\odot}$ & $0.56 \mathrm{M}_{\odot}$ \\
Primary Mass & $0.79 \mathrm{M}_{\odot} \ldots 1.44 \mathrm{M}_{\odot}$ & $0.53 \mathrm{M}_{\odot} \ldots 1.44 \mathrm{M}_{\odot}$ \\
Seconday Radius & $1.2 \mathrm{R}_{\odot} \ldots 1.1 \mathrm{R}_{\odot}$ & $0.92 \mathrm{R}_{\odot} \ldots 0.88 \mathrm{R}_{\odot}$ \\
Orbital Separation & $2.9 \mathrm{R}_{\odot} \ldots 3.2 \mathrm{R}_{\odot}$ & $2.4 \mathrm{R}_{\odot} \ldots 2.9 \mathrm{R}_{\odot}$ \\
Inclination & $6.8^{\circ} \ldots 8.3^{\circ}$ & $9.4^{\circ} \ldots 14.1^{\circ}$ \\
\hline
\end{tabular}

\section{References}

Cheng, F.H., Lin, D.N.C., 1989, Ap. J., 337, 432

Friend, M.T., Martin, J.S., Smith, R.C., Jones, D.H.P., 1990, MNRAS, 246, 654

Shafter, A.W., 1983, Ap. J., 267, 222

Szkody, P., Mattei, J.A., 1984, PASP, 96, 988 